\title{
Long-term Results after Total Thyroidectomy in Patients with Graves' Disease in Uzbekistan: Retrospective Study
}

\author{
${ }^{1}$ Said I Ismailov, ${ }^{2}$ Nusrat A Alimjanov, ${ }^{3}$ Bakhodir Kh Babakhanov, ${ }^{3}$ Murod M Rashitov, ${ }^{4}$ Alisher M Akbutaev \\ ${ }^{1}$ Professor and Director, The Center for the Scientific and Clinical Study of Endocrinology, Tashkent, Uzbekistan \\ ${ }^{2}$ Head, Department of Endocrine Surgery, The Center for the Scientific and Clinical Study of Endocrinology, Tashkent, Uzbekistan \\ ${ }^{3}$ Senior Scientific Worker, Department of Endocrine Surgery, The Center for the Scientific and Clinical Study of \\ Endocrinology, Tashkent, Uzbekistan \\ ${ }^{4}$ Junior Scientific Worker, Department of Endocrine Surgery, The Center for the Scientific and Clinical Study of \\ Endocrinology, Tashkent, Uzbekistan
}

Correspondence: Alisher M Akbutaev, Junior Scientific Worker, Department of Endocrine Surgery, The Center for the Scientific and Clinical Study of Endocrinology, 56 Mirzo Ulugbek Street, Mirzo Ulugbek, Tashkent-100125, Republic of Uzbekistan Phone: 998712622702, Fax: +998712622702, e-mail: alisheric@mail.ru

\section{ABSTRACT}

Subtotal thyroidectomy has been advocated as the standard treatment for Graves' disease (GD) because of the assumed lower risk of complications compared with total thyroidectomy, and also it provides the chance to avoid thyroxin therapy. The present study aims to examine our institutional experience with total thyroidectomy for GD. Patients were divided into two surgical treatment groups: Total thyroidectomy $(T T)(n=97)$ and total thyroidectomy with intraoperative thyroid autotransplantation (TTITA) $(n=74)$. TTITA performed in 74 patients. 0.5 to $2 \mathrm{gm}$ of thyroid tissue was cut into small pieces and autotransplanted into the forearm muscle of the patient. Postoperative complications included eight cases of RLN palsy, two patients had nerve paralysis, two patients underwent tracheostomy, transient hypoparathyroidism in 25 patients, permanent hypoparathyroidism in two cases, wound hemorrhage in two patients. TPOAb levels were increased in 9\% of patients with TT whereas in patients with TTITA TPOAb concentrations were elevated in $65 \%$ of patients at 3 months follow-up. TRAb in patients with TT were not detected while $20 \%$ patients undergone TTITA had high TRAb levels and $13.3 \%$ had terminal concentrations at 3 months follow-up. Serum TPOAb and TRAb were detected in none of the patients who underwent TT and TTITA at 1 , 3 and 5 years follow-up. Removal of all thyroid tissue offers the best chance of preventing recurrent hyperthyroidism and we saw no increase in postoperative complications in the TT group. We feel that TT is safe and superior for achieving the goal of treatment of Graves' disease.

Keywords: Graves' disease, Hyperthyroidism, Total thyroidectomy.

\section{INTRODUCTION}

Graves' disease is an autoimmune condition caused by thyroidstimulating antibodies that bind to the thyroid-stimulating hormone (TSH) receptors on thyroid follicular cells. It is characterized by symmetric diffuse goiter and hyperthyroidism, and is often accompanied by an infiltrative ophthalmopathy (GO) and, occasionally, by an infiltrative dermopathy. Graves' disease is the most common cause of hyperthyroidism. ${ }^{1}$ The annual incidences of GD are around 20 per 100,000 in Sweden and around 40 per 100,000 in the United States. It is four to six times more common in females and mostly occurs between 20 and 50 years of age. Current treatment for Graves' hyperthyroidism consists of antithyroid drugs, radioactive iodine and surgery. There is wide geographic variation in the choice of therapy, which in turn depends upon several factors, including the age of the patient, the severity of the disease, the size of the thyroid gland, any coexistent pathology, associated ophthalmopathy, contraindications to the use of RAI or antithyroid drugs, access to an experienced surgeon, tradition and the patient's preference, particularly in those female patients who are or want to become pregnant. ${ }^{2}$ Subtotal thyroidectomy has been advocated as the standard treatment for Graves' disease because of the assumed lower risk of complications compared with total thyroidectomy, and also because it provides the chance to avoid thyroxin therapy. However, the long-term results of subtotal thyroidectomy are not as good as originally thought, so now total thyroidectomy is being increasingly adopted for patients requiring surgical treatment for Graves' disease, based on a comparable surgical risk and the lack of recurrence as well as the questionable ability of subtotal thyroidectomy to maintain euthyroidism. We conducted this study to analyze the long-term results of total thyroidectomy for Graves' disease in a series of patients with reference to relevant literature. More recently, several investigators have suggested that total thyroidectomy should become the preferred procedure in the surgical management of patients with Graves' disease $\mathrm{e}^{4-7}$ based on several factors, the most important being the relative safety of total thyroidectomy in the hands of experienced surgeons. 
The present study aims to examine our institutional experience with thyroidectomy for Graves' disease with particular emphasis on the indications for surgery, procedures performed and their associated complications and long-term outcomes.

\section{MATERIALS AND METHODS}

Patient's medical records who underwent total thyroidectomy (TT) and total thyroidectomy with intraoperative thyroid autotransplantation (TTITA) in our department for the treatment of Graves' disease between 2000 and 2005 were retrospectively reviewed to obtain clinical data, including the indications for surgery, preoperative preparation, extent of surgery, postoperative course, morbidity, mortality. All operations were performed by one dedicated endocrine surgeon after obtaining informed consent from the patients. Patients were divided into two surgical treatment groups: Total thyroidectomy ( $\mathrm{n}=97)$, and total thyroidectomy with intraoperative thyroid autotransplantation $(n=74)$ (Fig. 1). Patients comprised 44 men with mean age of 37 (range, 20-55) and 127 women with mean age of 36 years (range, 1556). Eleven patients were lost at follow-up. The indication(s) for surgery was determined. The rate of recurrence of Graves' disease requiring additional medical therapy was calculated for each group. Routine antithyroid drugs were given to achieve an euthyroid state and $\beta$-blockers were given for symptom control. Lugol solution was given prior to surgery. The parathyroid glands were visualized and preserved. Intraoperative autotransplantation of thyroid tissue was carried out in 74 patients in order to prevent postoperative hypothyroidism. Approximately, 0.5 to 2 gm of thyroid tissue was cut into small pieces and autotransplanted into the forearm muscle of each patient. Patients were followed up for early and late complications. Routine postoperative serum calcium levels were measured, and calcium supplementation was given when the calcium level was below normal, or if the patient was symptomatic. Thyroxin was given to achieve euthyroidism in patients who underwent TT.

Surgical complications included transient and permanent hypoparathyroidism, transient and permanent recurrent

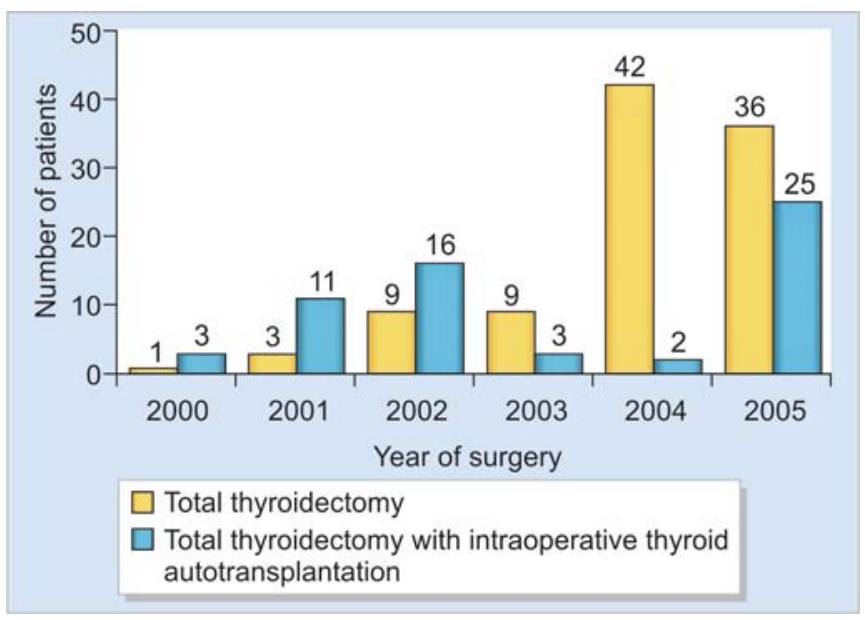

Fig. 1: Number of patients according to the years of surgery laryngeal nerve paralysis and wound hemorrhage. Graves' disease was diagnosed clinically by thyrotoxicosis and diffuse goiter with or without ocular findings. Thyrotropin receptor antibody (TRAb) and antithyroid peroxidase antibody (TPOAb) were measured using Immunotech a.s. kits (Czech Republic). Follow-up information was obtained by contacting patients.

\section{RESULTS}

Patients were evaluated for postoperative complications (Table 1) which included eight cases of recurrent laryngeal nerve (RLN) palsy, two patients had nerve paralysis, two patients underwent tracheostomy. Transient hypoparathyroidism developed in 25 patients while permanent hypoparathyroidism was seen in two cases. Transient hypoparathyroidism regressed after 1 month after calcium and vitamin D supplementation. Wound hemorrhage developed in two patients. Postoperative hypothyroidism was seen in all patients. It should be noted that postoperative hypothyroidism in patients with TTITA developed after 6 months following surgery and they needed thyroid hormones replacement as well as all other patients, hyperthyroidism recurrence developed in none of the patients.

Antithyroid peroxidase antibody levels were increased in $9 \%$ of patients with total thyroidectomy whereas in patients who underwent TTITA TPOAb concentrations were elevated in $65 \%$ of patients at 3 months follow-up (Fig. 2).

Thyrotropin receptor antibody in patients with TT were not detected whereas 20\% patients undergone TTITA had high TRAb levels and $13.3 \%$ had terminal concentrations at 3 months follow-up (Fig. 3).

Table 1: Complications of surgery

\begin{tabular}{lrr}
\hline & Number & $\%$ \\
\hline Permanent nerve paralysis & 2 & 1.2 \\
Nerve palsy & 8 & 4.7 \\
Permanent hypoparathyroidism & 2 & 1.2 \\
Transient hypoparathyroidism & 25 & 14.6 \\
Postoperative hemorrhage & 2 & 1.2 \\
Tracheostomy & 2 & 1.2 \\
\hline
\end{tabular}

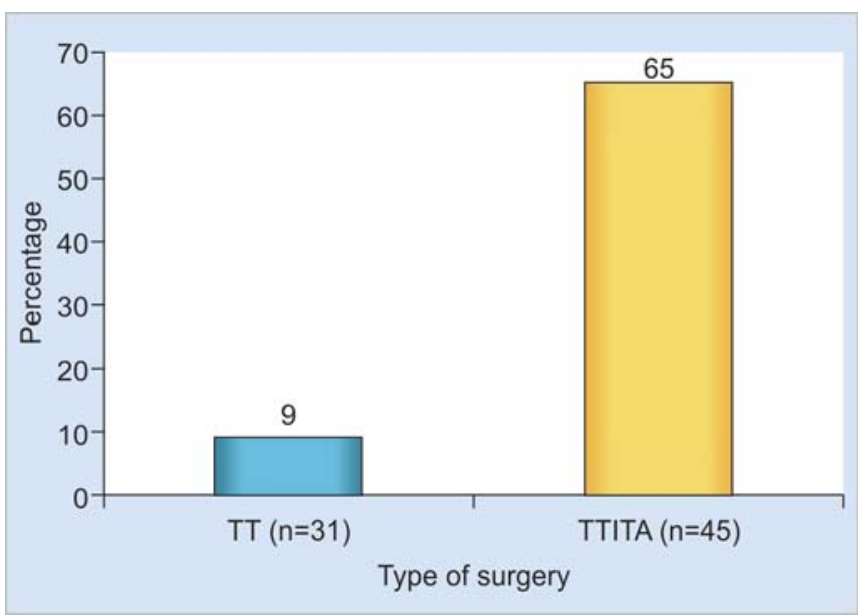

Fig. 2: TPOAb levels following surgery 


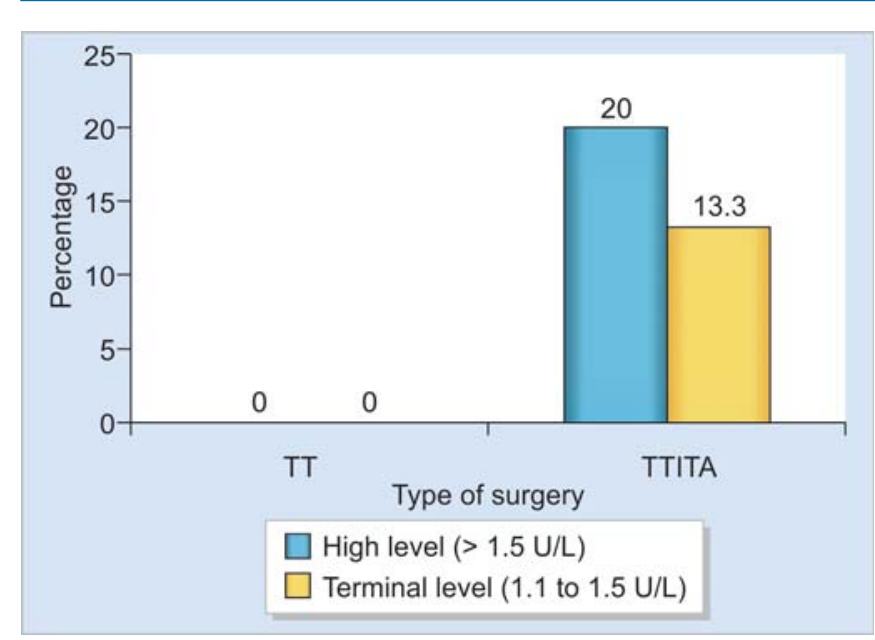

Fig. 3: TRAb levels following surgery

The laboratory data of patients who had total thyroidectomy and total thyroidectomy with intraoperative thyroid autotransplantation during 2000 to 2005 are shown in Table 2.

\section{DISCUSSION}

The goal of treatment for Graves' disease is to control the hyperthyroidism and restore euthyroidism. There are three major treatment modalities available for Graves' disease, and each option has its advantages and potential problems or side effects. When ${ }^{131}$ I radioablation was introduced, it was thought to be safer and less expensive than surgery; however, in the modern era of thyroid surgery, this argument must be revised. With effective drugs to treat the hyperthyroidism state preoperatively, such as propylthiouracil or thiamazole, iodine, propranolol and modern techniques, surgery is now safe for hyperthyroidism. ${ }^{3}$ The optimal definitive management of patients with Graves' disease remains a matter of debate and patients should be treated selectively. Several studies have examined the risks and benefits of various therapeutic options, and various factors need to be taken into consideration prior to advocating one approach over another. Torring et $\mathrm{al}^{8}$ performed a prospective randomized trial comparing antithyroid drugs, surgery or RAI and found that, although all treatments normalized hormone levels within 6 weeks, thyroidectomy was associated with the lowest relapse rates. RAI therapy has been shown to be associated with a slightly increased long-term risk of nodular goiter, thyroid cancer, hyperparathyroidism, unexplained increase in overall and cardiovascular mortality rates compared to the general population. ${ }^{9,10}$ In general, thyroidectomy is the preferred treatment option when RAI is contraindicated, as in patients who have confirmed thyroid malignancy, suspicious thyroid nodules, are young, pregnant or desire to conceive soon after treatment, have allergies to antithyroid medications, have large goiters causing compressive symptoms or those who are reluctant to undergo RAI therapy. ${ }^{11}$ Relative indications for thyroidectomy include patients particularly smokers with moderate to severe Graves' ophthalmopathy, those desiring rapid control of hyperthyroidism with a chance of being euthyroid and those demonstrating poor compliance to antithyroid medications.

In the present study, hyperthyroidism relapse developed in none of the patients after TT and TTITA, while hypothyroidism developed in $100 \%$ of cases which was successfully corrected by levothyroxine replacement. We also performed immune state evaluation in our patients. Latter showed no definite titers of TPOAb and TRAb (the main marker for Graves' hyperthyroidism) in patients who undergone total thyroidectomy, whereas in patients after total thyroidectomy with intraoperative thyroid autotransplantation TPO and TSH-R antibodies titers levels were high in 3 months time. However, in one year time after surgery, TPOAb and TRAb titers were not detected in these patients verifying that the implanted thyroid tissues were not alive and not functioning. Shimizu $\mathrm{K}$ et $\mathrm{al}^{12}$ and Okamoto $\mathrm{T}$ et $\mathrm{al}^{13}$ in their studies performed subtotal thyroidectomy with cryopreserved thyroid autotransplantation with a small number of patients and Shimizu $\mathrm{K}$ et $\mathrm{al}^{12}$ did not follow patients for a longer periods of time. The studies performed by Palit TK et al, ${ }^{4}$ Mittendorf EA et al, ${ }^{5}$ Torring $\mathrm{O}$ et al, ${ }^{8}$ Alsanea $\mathrm{O}$ et al ${ }^{11}$ Soreide JA et al, ${ }^{14}$ Witte et al, ${ }^{15}$ Sugino K et al, ${ }^{16} \mathrm{Ku}$ et al, ${ }^{17}$ demonstrated the superiority of total thyroidectomy in the surgical treatment of Graves' disease as: (1) no recurrent disease, (2) improvement of GO, (3) the rate of postoperative complications (RLN palsy, hypoparathyroidism, bleeding) do not increase after total thyroidectomy. The retrospective study performed in our center ${ }^{18}$ where patients were divided into two surgical treatment groups, including subtotal thyroidectomy and subtotal thyroidectomy with intraoperative thyroid autotransplantation, which showed that hyperthyroidism recurrence developed in $67 \%$ of patients in 15 to 20 years after surgery and was not correlated with thyroid autotransplantation whereas in present study no patients developed hyperthyroidism relapse. To summarize: (1) As we are aiming to reach hypothyroid state in hyperthyroid patients, (2) total thyroidectomy excludes

Table 2: Laboratory data after surgery

\begin{tabular}{|c|c|c|c|c|c|}
\hline \multirow[t]{2}{*}{ Group } & & \multicolumn{4}{|c|}{ Period of follow-up (Years) } \\
\hline & & 0.25 & 1 & 3 & 5 \\
\hline \multirow[t]{2}{*}{ Total thyroidectomy } & TPOAb detected & - & - & - & - \\
\hline & TRAb detected & - & - & - & 一 \\
\hline Total thyroidectomy with & TPOAb detected & Terminal to high level ${ }^{\mathrm{a}}$ & - & - & 一 \\
\hline $\begin{array}{l}\text { intraoperative thyroid } \\
\text { autotransplantation }\end{array}$ & TRAb detected & Terminal to high level ${ }^{a}$ & - & - & - \\
\hline
\end{tabular}

aHigh level (> $1.5 \mathrm{U} / \mathrm{L})$; Terminal level (1.1 to $1.5 \mathrm{U} / \mathrm{L})$ 
hyperthyroidism recurrence and (3) thyroid autotransplantation does not prevent hypothyroidism and thyrotoxicosis may recur. We came to conclusion that patients with Graves' disease should undergo total thyroidectomy to reach better treatment goals.

\section{CONCLUSION}

Total thyroidectomy offers a 100\% chance for cure of hyperthyroidism with a $0 \%$ recurrence rate. We believe that the primary goal of surgical therapy of Graves' disease is avoidance of recurrence rather than attainment of euthyroidism, particularly considering the indications for surgery in various series of patients, including our own. Total thyroidectomy can be safely performed by experienced surgeons and obviates the problems associated with lesser resections. Therefore, total thyroidectomy appears to be the preferred surgical approach for most patients with Graves' disease when it can be done with few complications.

\section{REFERENCES}

1. Tunbridge WM, Evered DC, Hall R, Appleton D, et al. The spectrum of thyroid disease in a community: The whickham survey. Clin Endocrinol 1977;7:481-93.

2. Claudio Casella, Giacomo Pata, Riccardo Nascimbeni, et al. Does extralaryngeal branching have an impact on the rate of postoperative transient or permanent recurrent laryngeal nerve palsy? World Journal of Surgery 2009;33(2):261-65.

3. Palit TK, Miller CC, Miltenburg DM. The efficacy of thyroidectomy for Graves' disease: A meta-analysis. J Surg Res 2000;90:161-65.

4. Mishra A, Agarwal A, Agarwal G, et al. Total thyroidectomy for benign thyroid disorders in an endemic region. World J Surg 2001;25:307-10.

5. Mittendorf EA, McHenry CR. Thyroidectomy for selected patients with hypotoxicosis. Arch Otolaryngol Head Neck Surg 2001;127:61-65.

6. Barakate MS, Agarwal G, Reeve TS, et al. Total thyroidectomy is now the preferred option for the surgical management of Graves’ disease. ANZ J Surg 2002;72:321-24.
7. Friguglietti CU, Lin CS, Kulcsar MA. Total thyroidectomy for benign thyroid disease. Laryngoscope 2003;113:1820-26.

8. Torring O, Tallstedt L, Wallin G, et al. Graves' hyperthyroidism: Treatment with antithyroid drugs, surgery, or radioiodine: A prospective, randomized study. Thyroid study group. J Clin Endocrinol Metab 1996;81:2986-93.

9. Franklyn JA, Maisonneuve P, Sheppard MC, et al. Mortality after the treatment of hyperthyroidism with radioactive iodine. N Engl J Med 1998;338:712-18.

10. Ron E, Doody MM, Becker DV, et al. Cancer mortality following treatment for adult hyperthyroidism. Cooperative Thyrotoxicosis Therapy Follow-up Study Group. JAMA 1998;280:347-55.

11. Alsanea O, Clark OH. Treatment of Graves' disease: The advantages of surgery. Endocrinol Metab Clin North Am 2000;29:321-37.

12. Shimizu K, Kumita S, Kitamura Y, et al. Trial of autotransplantation of cryopreserved thyroid tissue for postoperative hypothyroidism in patients with Graves' disease. Am Coll Surg 2002;194:14-22.

13. Okamoto T, Fujimoto Y, Obara T, et al. Trial of thyroid autotransplantation in patients with Graves' disease whose remnant thyroid has unintentionally been made too small at subtotal thyroidectomy. Endocrinol Jpn Feb 1990;37(1):95-101.

14. Soreide JA, Jon A van Heerden, Lo CY, et al. Surgical treatment of Graves' disease in patients younger than 18 years. World J Surg 1996;20:794-800.

15. Witte J, Goretzki PE, Dotzenrath C, et al. Surgery for Graves' disease-Total versus subtotal thyroidectomy: Results of a prospective randomized trial. World J Surg 2000;24:1303-11.

16. Sugino K, Mimura T, Ozaki O, et al. Preoperative change of thyroid stimulating hormone receptor antibody level: Possible marker for predicting recurrent hyperthyroidism in patients with Graves' disease after subtotal thyroidectomy. World J Surg 1996;20:801-07.

17. Ku CF, Lo CY, Chan WF, et al. Total thyroidectomy replaces subtotal thyroidectomy as the preferred surgical treatment for Graves’ disease. ANZ J Surg 2005;75:528-31.

18. Ismailov SI, Alimdjanov NA, Babakhanov BKh, et al. Longterm results after subtotal thyroidectomy with and without intraoperative thyroid autotransplantation in patients with Graves’ disease. Uzb Surg 2009;4:17-20. 